\title{
Semi Markov Decision Processes for Service Facility Systems with Perishable Inventory
}

\author{
R. Satheesh Kumar \\ PSNA College of Engineering and Technology, \\ Dindigul, \\ Department of Mathematical Sciences,
}

\author{
C.Elango \\ Cardamom Planters' Association college, \\ Bodinayakanur. \\ Department of MathematicalScience
}

\begin{abstract}
We consider a service facility system with perishable inventory with finite number of servers. Each server having one perishable item for providing service. The arrival of a customer at the system according to independent Poisson Processes with rate $\lambda$ through a single channel. The service time of the customer is exponentially distributed with mean $1 / \mu$ and the item in stock has exponential life time with perishability rate $\gamma(>0)$. The free servers are occupied by the new customer, entering for service. The maximum level of item in stock is $S(=c)>0$. The replenishment of inventory is instantaneous, when the inventory level comes to zero. Customers can be rejected from the system only when all the servers are busy that is, there is no waiting line. Transition probabilities are obtained from the two dimensional process. The problem is modeled as a Semi Markov decision process and we use the modified Value Iteration algorithm to obtain the minimum average loss rate.
\end{abstract}

Key words: Service facility system, Perishable inventory, Semi Markov decision processes, Modified Value iteration, Data transformation.

\section{INTRODUCTION}

In most of the perishable inventory models, the inventory is depleted at a rate equal to the demand rate and perishable rate. But in service facility, we have a different situation when there are customers request for service. Thus the depletion of inventory depends on service rate and perishability rate. Examples of perishable inventory at service facilities include units of blood necessary for surgery, maintain the fruit stock in juice factory and maintain the milk stock in foot stalls which sell milk made products.

Berman et. al(1993)considered an inventory management system with a service facility using one item of the inventory for each service. They assumed that both the demand and service times are deterministic and that queues can occur only during the stock-outs. They determined the optimal order quantity that minimizes the total expected cost rate.

Berman and Kim(1999) analyzed a problem in a stochastic environment where the customers arrive at a service facility according to a Poisson Process. The service times are exponentially distributed with the mean inter-arrival time is assumed to be larger than the mean service time. The optimal policy in their paper is derived given that the order quantity is known. A logically related model has been studied by He et. al(1998), who analysed a Markovian inventory-production system.

Berman and Sapna(2000) studied an inventory control problem at a service facility requiring one item of the inventory and assumed Poisson arrivals, arbitrarily distributed service times and zero lead times with a waiting room of finite capacity. Under a specified cost structure, the optimal ordering quantity that minimizes the long-run expected cost rate has been derived.

Elango(2001) considered a Markovian inventory system at a service facility with service time having an exponential distribution with the parameter depending on the number of waiting customers and zero lead time. Arivarignan et. al(2002) extended this model to include an exponential lead time. Perumal and Arivarignan(2002) considered a Markovian inventory system with a waiting room of infinite capacity. Arivarignan and Sivakumar(2003) considered an inventory system with arbitrary distribution for inter-occurrence time of demands, exponential service time and exponential lead time.

Eungab Kim(2005).[8] treat an inventory control problem in a facility that provides a single type of service for customers. Items used in service are supplied by an outside supplier. To incorporate lost sales due to service delay into the inventory control, they model a queuing system with finite waiting room and non-instantaneous replenishment process and examine the impact of finite buffer on replenishment policies.

Maria E. Mayorga, Hyun-Soo Ahn(2006).[12] considered a multi class make-to-stock system served by a single server with adjustable capacity(service rate). At any point in time, the decision-maker must determine the capacity level, make a production decision(ie.,whether to produce an item to stock or to satisfy a back-order), and make a rationing decision(ie.,whether to satisfy a new order from stock or place it on back-order). They characterize the structure of optimal capacity adjustment, production, and stock rational policy for both finite and infinite-horizon problems. He find that the optimal policy is monotone in current inventory and backorder levels, and characterize its properties.

In this article, the system has $C$ identical servers and each customer occupying one free server for service. The service facility system attached with perishable inventory and one item is utilised for each service. The replenishment of inventory is assumed to be instantaneous (ie., $(0, S)$ Policy). The maximum level of inventory is $S(=c)>0$ During the time of service, the depletion of the inventory is considered by perish and service completion of the customers. Decision is taken to accept the customer or reject the customer for service. Assume that there is no cost for acceptance of the customer and the cost will be incurred for rejection.

This paper is organised as follows. Section 2, deals with the formulation of the problem. Section 3, which contains the analysis part of the problem forms the core part of the paper and we present some numerical results. Direction for 
future research and suggestions are provided in the last section 4.

\section{PROBLEM FORMULATION}

Consider a Service facility system with perishable inventory which having $c$ identical servers. Due to perishability, we keep the maximum inventory level at $S(=c>0)$ ( ie each server can utilize one item for service at a time). Customers arrive for service according to a Poisson Process with rate $\lambda(>0)$ and the service time is exponentially distributed with mean $\frac{1}{\mu}(>0)$. The item in stock has exponential life time with parameter $\gamma(>0)$.

\section{Formulation with fictitious decision epochs}

In such a formulation, the vectors $\left(p_{i j}(a),(i, j) \in I\right)$ of one step transition probabilities have many non- zero entries. In our specific problem, this difficulty can be circumvented by including the service completion epochs and depletion epochs of perished item as fictitious decision epochs in addition to the real decision epochs, being the arrival epoch of customers. By doing so, a transition from any state is always to one of at most four neighboring states. In the approach with fictitious decision epochs, we take the state space:

$I=\{(q, i, k) / q, i=0,1,2, \ldots c ; q, i \leq c ; k=0,1\}$ State

$(q, i, k)$ with $\mathrm{k}=1$ corresponds to the situation states that the customer entering point for service in the system. The auxiliary state $(q, i, 0)$ corresponds to the situation in which the item is depleted by completion of service or perish of the item. Note that the customer arrival and the service completions are not relevant.

For the states $(q, i, k)$ with $\mathrm{k}=1$, the possible actions are denoted by,

$a=\left\{\begin{array}{l}0-\text { reject the arriving customer at }(q, i, 1) \\ 1-\text { accept the arriving customer at }(q, i, 1)\end{array}\right.$

with the stipulation that $a=0$ is only feasible decision, when $q=i=c$. The fictitious decision of leaving the customers from the system and depletion of the item from inventory alone in the state $s=(q, i, 0)$ is also denoted by $a=0$.

Further the transition probabilities are extremely easy to specify, because of the fact that $\min \left(X_{1}, X_{2}\right)$ is exponentially distributed with mean $\frac{1}{a_{1}+\alpha_{2}}$ and $\frac{1}{\alpha_{1}+\alpha_{2}}$

$P\left\{X_{1}<X_{2}\right\}=\frac{\alpha_{1}}{\alpha_{1}+\alpha_{2}}$, when $X_{1}$ and $X_{2}$ are independent random variables having exponentially distributed with respective means $\frac{1}{\alpha_{1}}$ and $\frac{1}{\alpha_{2}}$

The total event rate is

$$
v(q, i)= \begin{cases}\lambda+i \gamma+q \mu, & q \leq i \\ \lambda+i(\gamma+\mu), & q>i\end{cases}
$$

\section{VALUE ITERATION}

For the Semi Markov decision model the formulation of a value-iteration algorithm is not straight forward. However, by the data transformation method, we can convert the Semi-Markov decision model into a discrete -time Markov decision model such that both models have the same average cost for each stationary policy. In the discrete-time model it is no restriction to assume that all $\bar{c}_{s}(a)=\frac{c_{s}(a)}{\tau_{s}(a)}$ are positive. Otherwise add a sufficiently large positive constant to each $\bar{c}_{s}(a)$.

\section{Data Transformation method}

First chose a number $\tau$ with $0<\tau \leq \min _{(s, a)} \tau_{s}(a)$. where $s=(q, i, k)$. Consider now, the discrete time Markov decision model whose basic elements are given by,

$$
\begin{aligned}
& \bar{I}=I, \text { and } \bar{A}(s)=A(s), s \in \bar{I} . \\
& \bar{c}_{s}(a)=\frac{c_{s}(a)}{\tau_{s}(a)}, a \in \bar{A}(s) \text { and } s \in \bar{I} .
\end{aligned}
$$

$\bar{p}_{s v}(a)=\left\{\begin{array}{lll}{\left[\frac{\tau}{\tau_{s}(a)}\right] \cdot p_{s v}(a),} & s \neq v, \quad a \in \bar{A}(s) \text { and } s \in \bar{I} \\ {\left[\frac{\tau}{\tau_{s}(a)}\right] \cdot p_{s v}(a)+\left[1-\frac{\tau}{\tau_{s}(a)}\right],} & s=v, \quad a \in \bar{A}(s) \text { and } s \in \bar{I}\end{array}\right.$

the choice of $\tau$ in the algorithm, it is recommended to take $\tau=\min _{(s, a)} \tau_{s}(a)$ when the embedded Markov chains $\left\{X_{n}\right\}$ in the Semi Markov model are aperiodic; otherwise $\tau=\frac{1}{2} \min \tau_{s}(a)$ is a reasonable choice.

Then for action $a=0$ in state $s=(q, i, k)$

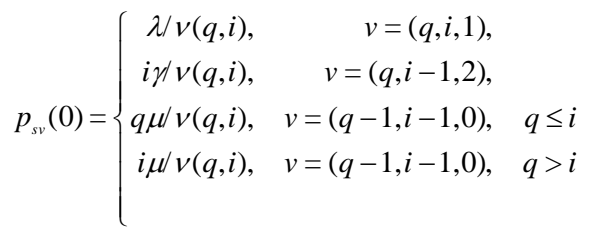

$$
\text { and } \tau_{s}(0)=\frac{1}{v(q, i)} \text {. }
$$

For action $a=1$ in state $s=(q, i, 1)$

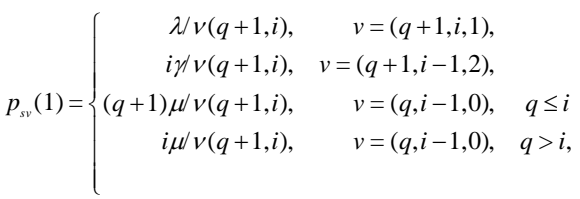


and $\tau_{s}(1)=\frac{1}{v(q+1, i)}$

Finally the one step expected costs $c_{s}(a)$ are simply given by,

$c_{s}(a)= \begin{cases}1, & s=(q, i, 1) \text { and } a=0 \\ 0, & \text { otherwise, }\end{cases}$

Now, having specified the basic elements of the SemiMarkov decision model, we are in a position to formulate the value iteration algorithm for the computation of a(nearly) optimal acceptance rule. In the data transformation we take,

$\tau= \begin{cases}1 / \lambda+i \gamma+q \mu, & q \leq i \\ 1 / \lambda+i(\gamma+\mu), & q>i\end{cases}$

Using the above specifications, the value iteration scheme becomes quite simple for the allocation problem. Note that the expressions for the one-step transition times $\tau_{s}(a)$ and the one-step transition probabilities $p_{s v}(a)$ have common denomination and so the ratio $p_{s v}(a) / \tau_{s}(a)$ has a very simple form. In specifying the value iteration scheme, we distinguish between the auxiliary states $(q, i, 0)$ and the other states.

\section{Convergence of the bounds}

In value iteration for discrete time Markov decision problem, the lower and upper bounds $m_{n}$ and $M_{n}$ converge to the same limit so that the algorithm will be stopped after finitely many iterations only, if a certain aperiodicity condition is satisfied. In general $m_{n}$ and $M_{n}$ need not have the same limit, as the following example demonstrates. Consider the trivial Markov decision problem with two states 1 and 2 and a single action $a_{0}$ in each state. The one-step costs and the one-step transition probabilities are given by $c_{1}\left(a_{0}\right)=1, c_{2}\left(a_{0}\right)=0, p_{12}\left(a_{0}\right)=p_{21}\left(a_{0}\right)=1$ and $p_{11}\left(a_{0}\right)=p_{22}\left(a_{0}\right)=0$. Then the system cycles between the states 1 and 2 . It is easily verified that $V_{2 k}(1)=V_{2 k}(2)=k, V_{2 k-1}(1)=k \quad$ and $\quad V_{2 k-1}(2)=k-1$ for all $k \geq 1$. Hence $m_{n}=0$ and $M_{n}=1$ for all $\mathrm{n}$, implying that the sequences $m_{n}$ and $M_{n}$ have different limits. The reason for the oscillating behavior of $V_{n}(i)-V_{n-1}(i)$ is the periodicity of the Markov chain describing the state of the system. The next theorem gives sufficient conditions for the convergence of the valueiteration algorithm.

\section{Theorem}

Suppose the weak unichain assumption holds and that for each average cost optimal stationary policy the associated Markov chain $X_{n}$ is aperiodic. Then there are finite constants $\alpha>0$ and $0<\beta<1$ such that $\left|M_{n}-m_{n}\right| \leq \alpha \beta^{n}, \quad n \geq 1 . \quad$ In particular, $\lim _{n \rightarrow \infty} M_{n}=\lim _{n \rightarrow \infty} m_{n}=g^{*}$. For the proof of the theorem for the special case ofStrong aperiodicity assumption.[(i) for each stationary policy $\mathrm{R}$ the associated Markov chain $X_{n}$ has no two disjoint closed sets; (ii) $p_{i i}(a)>0$ for all $i \in I$ and $\left.a \in A(i)\right]$ see Tijms (pp-270, 2003).

From the above result,

$\left.M_{n}-m_{n} \leq(1-\rho)\left(M_{n-N}\right)-m_{n-N}\right) \quad$ where $\rho=\frac{\lambda}{c \mu}$.

it was shown that $M_{n}-m_{n}, n \geq 1$ is non- increasing. Thus we find that

$M_{n}-m_{n} \leq(1-\rho)^{(n / N)}\left(M_{0}-m_{0}\right), \quad n \geq 1 . \quad$ implying the desired result.

\section{Value iteration algorithm}

Step:0 Choose $V_{0}(s) \quad$ such that $0 \leq V_{0}(s) \leq \min \left\{c_{s}(a) / \tau_{s}(a)\right\}$ for all $s$. choose a number $\tau$ with $0<\tau \leq \min \left\{\tau_{s}(a)\right\}$.

Step:1 In the states $(q, i, 0)$ the only possible decision is to leave the system alone. Thus,

$V_{n}(q, i, 0)= \begin{cases}\tau \lambda V_{n-1}(q, i, 1)+\tau i \gamma V_{n-1}(q, i-1,0) \\ +\tau q \mu V_{n-1}(q-1, i-1,0), & \text { for } q \leq i \\ \tau \lambda V_{n-1}(q, i, 1)+\tau i \gamma V_{n-1}(q, i-1,0) & \text { Where } \\ +\tau i \mu V_{n-1}(q-1, i-1,0), & \text { for } q>i\end{cases}$
$V_{n-1}(q, i, 1)=0$ and $V_{n-1}(q, i, 0)=0$ when $q \leq 0$ or $i \leq 0$.

Then the action $\mathrm{a}=1$ for the states $(q, i, 1)$

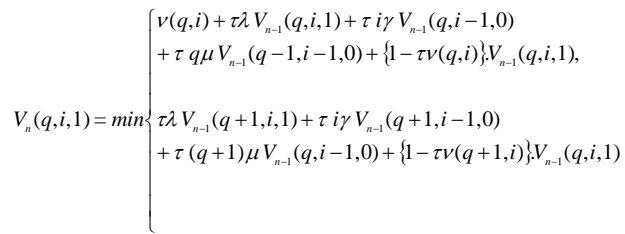

for $(q+1) \leq i$

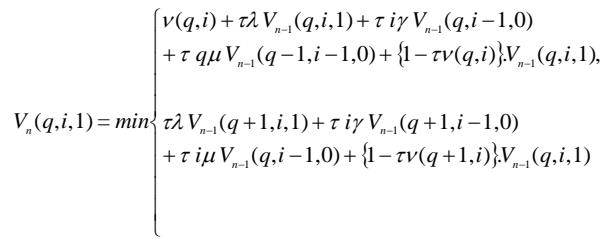

for $(q+1)>i$ 
step:2 Compute the bounds when $s=(q, i, 1)$

$$
\begin{aligned}
& m_{n}=\min \left\{V_{n}(s)-V_{n-1}(s)\right\} \\
& M_{n}=\max \left\{V_{n}(s)-V_{n-1}(s)\right\}
\end{aligned}
$$

\begin{tabular}{|c|c|c|c|c|c|c|c|}
\hline $\begin{array}{l}S . \\
N o\end{array}$ & $\lambda$ & $\mu$ & $\gamma$ & 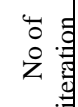 & $\mathrm{y}$ & Z & $g^{*}$ \\
\hline 1 & 6 & 10 & 1 & 49 & 1.5673 & 1.6503 & 1.6088 \\
\hline 2 & 5 & 9 & 1 & 53 & 1.2749 & 1.4240 & 1.3494 \\
\hline 3 & 5 & 10 & 1 & 57 & 1.2699 & 1.4540 & 1.3619 \\
\hline 4 & 6 & 10 & 2 & 59 & 1.3437 & 1.3581 & 1.3509 \\
\hline 5 & 4 & 8 & 1 & 63 & 1.0151 & 1.0483 & 1.0317 \\
\hline 6 & 4 & 9 & 1 & 65 & 1.0319 & 1.1054 & 1.0687 \\
\hline 7 & 5 & 10 & 2 & 76 & 0.7867 & 1.0151 & 0.9009 \\
\hline 8 & 4 & 8 & 2 & 85 & 0.4748 & 0.5109 & 0.4928 \\
\hline 9 & 4 & 10 & 2 & 94 & 0.5488 & 0.5663 & 0.5575 \\
\hline 10 & 4 & 9 & 2 & 104 & 0.3784 & 0.4252 & 0.4018 \\
\hline
\end{tabular}

$\begin{array}{llrr}\text { The } \quad \text { algorithm } & \text { is } \quad \text { stopped } & \text { when } \\ M_{n}-m_{n} \leq(1-\rho)\left(M_{n-N}-m_{n-N}\right) & \text { Here } & N=1 .\end{array}$

Then the algorithm stops after finitely many iterations, we can get the average lost rate $g^{*}$.

Numerical illustration is given below.

We consider that the number of servers in the system is $\mathrm{c}=4$.

$y=M_{n}-m_{n}$

$z=(1-\rho)\left(M_{n-2}-m_{n-2}\right)$

$g^{*}=y / 2$

\section{ACKNOWLEDGEMENTS}

We have considered a service facility system with perishable inventory, as a Markov decision problem. Value iteration is the procedure used to get optimal average cost for loss rate of the customers. The complexity of the problem is more when the lead time of the item procurement is constant or exponentially distributed. To the best of our knowledge this MDP method is used first time to control the inventory in service facility ( Berman, $\mathrm{O}$. and Sapna, K. P. considered control of service rates). In future research investigations of MDP in service facility systems to compute the lose rate of the customer whenever the lead time of the item procurement is exponentially distributed.

\section{REFERENCES}

[1] Arivaringan G, Elango C and Arumugam N. A continuous review perishable inventory control system at service facilities, Notable Publications Inc. USA(2002),pp.29-39.
[2] Arivaringan G, Sivakumar, B. Inventory system with renewal demands at service facilities. In: Srinivanan S.K and Vijayakumar A.(eds.) Stochastic Point Processes, ,Notable Publishing House, New Delhi, India(2003), pp.108-123.

[3] Berman O,Kaplan EH, and Shimshak DG. Deterministic approximations for inventory management at service facilities. IIE Transactions (1993)104, pp.98-104

[4] Berman O, Kim E. Stochastic inventory policies for inventory management at service facilities.Stochastic Models(1999) 15 , pp.695-718.

[5] Berman O, Sapna KP. Inventory management at service facilities for systems with arbitrarily distributed service times, Stochastic Models,(2000), pp. 16, 343-360.

[6] Berman O, Sapna KP. Optimal control of service for facilities holding inventory, Computers and Operations Research, (2001) 28, pp. 429-441.

[7] Elango C. Inventory system at service facilities, Ph.D Thesis,(2001) Madurai Kamaraj University, India.

[8] Eungab Kim. Optimal inventory replenishment policy for a queuing system with finite waiting room capacity,European Journal of Operational research(2005),161, No.1, pp.256-274.

[9] Howard RA. Dynamic Programming and Markov Processes.John Wiley and sons, Inc: New York; 1960.

[10] He, Q-M., Jewkes, E.M and Buzacott, J. An efficient algorithm for computing the optimal replenishment policy for an inventory-production system. In: Alfa, A. Chakravarthy S.(eds), Advances in matrix Analytic methods for ,Notable Publications, New Jersey, USA.(1998), pp.381-402.

[11] He, Q-M., Neuts, M.F. Markov chains with marked transitions. ,Stochastic Processes and their Applications(1998),74, pp.37-52.

[12] Maria E. Mayorya, Hyun-Soo Ahn., George Shanthikumar J. Optimal control of a make - to - stock system with adjustable service rate, Probability in Engineering and Information Sciences(2006),20, No.4, pp.609-634.

[13] Nahmias, S. Perishable inventory theory: A review. Operations Research,(1982),30 pp.680-708.

[14] Perumal, V and Arivarignan, G. A continuous review perishable inventory system at infinite capacity service facilities. ANJAC Journal of Science,(2002),1, pp.37-45.

[15] Puterman ML. Markov Decision Processes: Discrete Stochastic Dynamic Programming.John Wiley and sons, Inc: New York; 1994.

[16] Ross, S.M. Introduction to Probability Models, Harcourt Asia PTE Ltd, Singapore. 2000.

[17] Tijms HC. A first course in stochastic models,John Wiley and sons, Ltd: England; 2003. 